\title{
ARTICLE OPEN Individualized plasticity autograft mimic with efficient bioactivity inducing osteogenesis
}

Yan Wei ${ }^{1,2}$, Guixin Zhu' ${ }^{1}$, Zifan Zhao ${ }^{1}$, Chengcheng Yin ${ }^{1}$, Qin Zhao ${ }^{1}{ }^{1}$, Hudi Xu ${ }^{2}$, Jinyang Wang ${ }^{1}$, Jinglun Zhang ${ }^{1}$, Xiaoxin Zhang $^{2}$, Yufeng Zhang iD $^{1,2}$ and Haibin Xia ${ }^{1,2}$

Mineralized tissue regeneration is an important and challenging part of the field of tissue engineering and regeneration. At present, autograft harvest procedures may cause secondary trauma to patients, while bone scaffold materials lack osteogenic activity, resulting in a limited application. Loaded with osteogenic induction growth factor can improve the osteoinductive performance of bone graft, but the explosive release of growth factor may also cause side effects. In this study, we innovatively used platelet-rich fibrin (PRF)modified bone scaffolds (Bio-Oss') to replace autograft, and used cytokine (BMP-2) to enhance osteogenesis. Encouragingly, this mixture, which we named "Autograft Mimic (AGM)", has multiple functions and advantages. (1) The fiber network provided by PRF binds the entire bone scaffold together, thereby shaping the bone grafts and maintaining the space of the defect area. (2) The sustained release of BMP-2 from bone graft promoted bone regeneration continuously. (3) AGM recruited bone marrow mesenchymal stem cells (BMSCs) and promote their proliferation, migration, and osteogenic differentiation. Thus, AGM developed in this study can improve osteogenesis, and provide new guidance for the development of clinical bone grafts.

International Journal of Oral Science (2021)13:14

; https://doi.org/10.1038/s41368-021-00120-w

\section{INTRODUCTION}

Since the twenty-first century, several studies have focused on the morphological recovery of tumors, infections, biochemical disorders, skeletal dysplasia, and trauma caused by extensive tissue defects $^{1,2}$. Regeneration of hard tissues, including bone tissue, is important but challenging in tissue engineering and regeneration $^{3-5}$. Autograft exhibits excellent regeneration effect, but the second surgery area increased the risk of postoperative complications ${ }^{6}$. Besides, the absorption of autograft is relatively fast and hard to control ${ }^{7}$. The autogenous bone is also difficult to adjust to the proper shape of the bone defect, which limits its application ${ }^{8}$. Therefore, researchers and clinicians set their sights on bone substitutes, which are widely used clinically because of their abundant sources 9 . However, bone substitutes lack biological components, such as osteoblasts and growth factors, resulting in slow bone regeneration and poor osteoinductivity ${ }^{10}$. Moreover, the granular morphology of bone substitutes is not conducive to clinical operations, causing certain difficulties to fit the defect area ${ }^{11}$. Although new bone regeneration materials are continuously emerging, ideal biological materials that can solve the abovementioned problems remain lacking.

The combination of osteogenic inductive growth factors can improve the osteoinductive properties of bone grafts and stimulate mesenchymal stem cells around the bone grafts to differentiate into chondroblasts or osteoblasts and form new bone ${ }^{10}$. Common growth factors include transforming growth factor-beta (TGF- $\beta$ ), bone morphogenetic proteins (BMPs), fibroblast growth factors, insulin-like growth factors (IGFs), and platelet-derived growth factors (PDGFs). These growth factors interact with each other during fracture healing and mediate bone regeneration ${ }^{12-14}$. BMP-2 is currently the only Food and Drug Administration (FDA)-approved osteoinductive growth factor, widely used with the bone substitutes in clinical practice ${ }^{15}$. BMP-2, an osteogenic cytokine, induces bone formation by promoting the proliferation and osteogenic differentiation of mesenchymal stem cells, which secret mineralized matrix and collagen locally ${ }^{14,16,17}$. BMP-2 combined with bone substitutes exerts osteoinductive effects, and bone substitutes serve as its carrier and scaffold to provide ideal mechanical properties for improved osteogenic effects ${ }^{18-20}$.

Although the combination of BMP-2 and bone substitutes has the above advantages in osteoinduction, BMP-2 is released explosively during the clinical application, and its half-life in vivo is short ${ }^{21}$. Excessive concentrations of BMP-2 in the early stages of mineralization may also cause side effects, such as ectopic osteogenesis, osteolysis and sedimentation, bone cysts, local inflammatory reactions, traumatic injuries, postoperative fever, and hemorrhage, and even cancer ${ }^{15,22}$. Therefore, the focus of our research is finding a carrier that can safely and effectively release BMP-2 and improve the operability of bone substitutes.

Platelet-rich fibrin (PRF), the second-generation biomaterial obtained by centrifugation from autologous blood, is rich in platelets and leukocytes, functions as a fibrin scaffold and regulator of various cytokines ${ }^{23}$. Numerous scholars have conducted in-depth research on PRF, confirming the positive role of PRF in the regeneration of soft tissue and hard tissue defects ${ }^{24,25}$. Micropores are composed of the fibers within PRF and can function as scaffolds for cell migration, proliferation, and

\footnotetext{
${ }^{1}$ The State Key Laboratory Breeding Base of Basic Science of Stomatology (Hubei-MOST) \& Key Laboratory of Oral Biomedicine Ministry of Education, School \& Hospital of Stomatology, Wuhan University, Wuhan, China and ${ }^{2}$ Department of Oral Implantology, School and Hospital of Stomatology, Wuhan University, Wuhan, China Correspondence: Haibin Xia (xhaibin@whu.edu.cn)

These authors contributed equally: Yan Wei, Guixin Zhu
}

Received: 18 October 2020 Revised: 8 February 2021 Accepted: 2 March 2021

Published online: 12 April 2021 
a

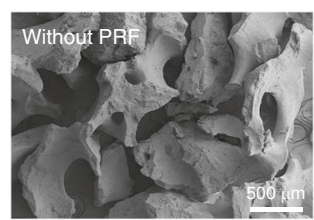

b

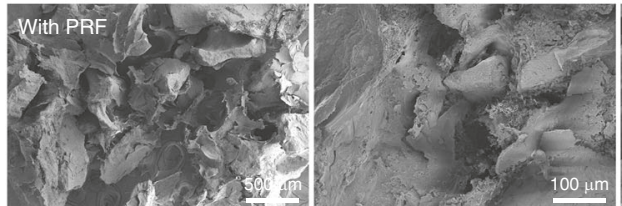

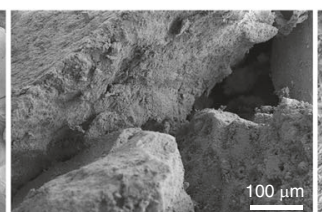
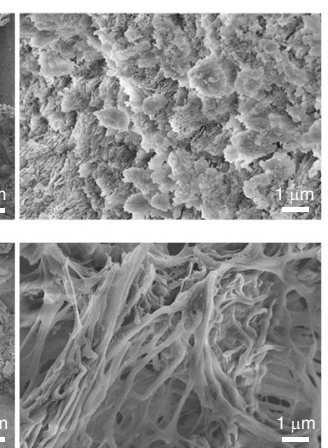

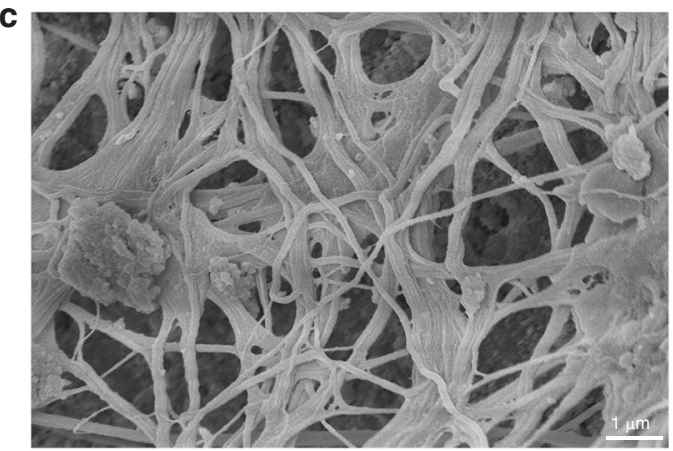

Fig. 1 Fiber networks formed on the surface of Bio-Oss ${ }^{\circledR}$ to giving plasticity for AGM. a, b SEM micrographs of surface morphology of bone grafts with or without PRF. c SEM micrograph of the fibrin mesh between Bio-Oss ${ }^{\circledR}$ granules
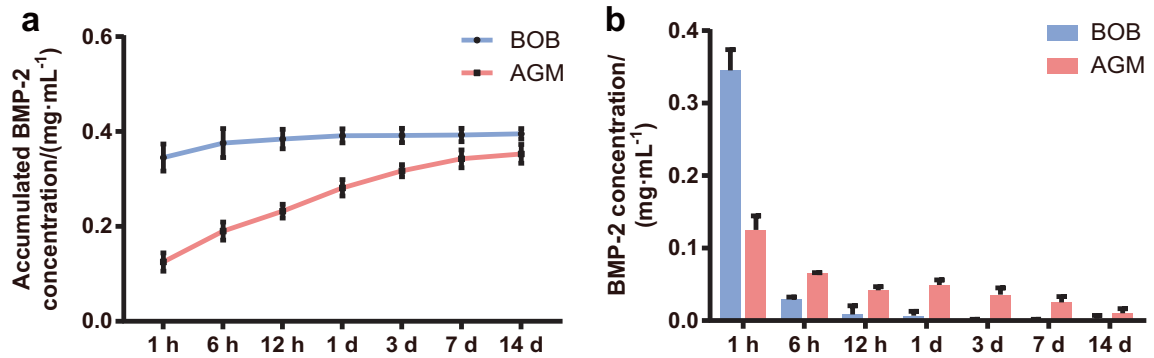

Fig. 2 BMP-2 sustained release from AGM. a Total accumulated BMP-2 released from BOB and AGM over 14 days $(n=3)$. b BMP-2 release from BOB and AGM at each time point over 14 days $(n=3)$

differentiation as well as for delivery of growth factors ${ }^{26}$. Many studies have shown that PRF can gradually release its cytokines and cells ${ }^{27,28}$. It was shown that one of the reasons may be that cytokines are trapped in the fiber network, and even combine with the fiber and platelets in $\mathrm{PRF}^{29}$. With the fibrin network gradually destroy and remodel, the cytokines are released slowly. Also, PRF can protect growth factors from protease hydrolysis ${ }^{30}$. With the concept of low-speed centrifugation in recent years, modified liquid PRF demonstrates improved release of growth factors and abundance of leukocytes and platelets ${ }^{23}$. However, the endogenous growth facts release is far from enough to promote bone regeneration. Therefore, we supplemented FDA-approved BMP-2 to optimize the effect of bone regeneration ${ }^{31,32}$.

Here, we constructed an improved bone graft with osteoconductivity, osteoinductivity, enhanced mechanical properties, and good biocompatibility by using the bone substitute Bio-Oss, the growth factor BMP-2, and liquid PRF. The new bone graft Bio-Oss / PRF/BMP-2, named "autograft mimic (AGM)", promoted the proliferation, migration, and mineralization of BMSCs in vitro and considerably improved bone regeneration in vivo. AGM overcomes most of the limitations of current bone substitutes and is capable of local sustained release of BMP-2 and its cytokines, thereby allowing BMP-2 to promote tissue regeneration continuously during the healing of bone defect and considerably enhance osteogenesis.

\section{RESULTS}

Morphological characteristics of grafts

Liquid PRF forms with lower centrifugal force and shorter centrifugal time than traditional PRF manufacturing methods ${ }^{33}$. The results suggest that PRF converts the morphology of Bio-Oss from granules into blocks and facilitates clinical operability (Fig. 1 and Supplement). Blood was collected into special plastic centrifuge tubes that do not contain anticoagulants. After centrifugation, the collected blood was divided into two layers: the upper PRF layer and the lower red blood cell layer
(Supplement 1A). After mixing with PRF, Bio-Oss ${ }^{\oplus}$ changed greatly from a conventional granular form into a block that can remains stable during operation (Supplement 1B). The morphology of the bone grafts was observed by scanning electron microscopy (SEM). Bio-Oss without PRF was dispersive at low-power objectives of $\times 40$ (left) and $\times 200$ (middle). When mixed with PRF, Bio-Oss ${ }^{\circledast}$ was clustered with the filling of PRF. SEM under a high-power objective of $\times 10000$ (right) illustrated that the surface of Bio-Oss ${ }^{\circledast}$ without PRF showed a typical hydroxyapatite structure, whereas that of Bio-Oss with PRF contained a large number of fiber bundles (Fig. 1a, b). And the fibrin mesh is obvious between Bio-Oss ${ }^{\oplus}$ granules (Fig. 1c).

Sustained release of BMP-2 in vitro

The three-dimensional (3D) network of fibers connecting bone substitutes was observed by SEM. Micropores composed of those fibers can serve as scaffolds for cell proliferation, migration, and differentiation likewise for delivery of growth factors ${ }^{34}$. Therefore, its controlled release characteristics were evaluated and quantified by measuring the accumulated BMP-2 concentration and the BMP-2 concentration at the required time point (Fig. 2a, b). In materials without PRF, the release of BMP-2 was explosive within the first $6 \mathrm{~h}$, reached a peak quickly, and then almost stopped. (Fig. 2a). By contrast, in the grafts with PRF, the release of BMP-2 was more gradual and persistent, even maintained a small amount of release up to 14 days (Fig. 2a, b).

AGM promotes BMSCs proliferation and migration in vitro The effects of BMP-2 and PRF on cell proliferation and migration have been extensively studied in previous studies ${ }^{35-38}$. To further confirm the biological properties of the four different bone grafts, we co-cultured BMSCs harvested from a rat with the four samples and determined cell proliferation by the CCK- 8 assay (Fig. 3a). Compared with the control group using Bio-Oss scaffolds alone, the BOP group significantly increased the number of cells on days 3 and 5 . However, a significant decrease in proliferation ability was observed in the BOB group. Interestingly, there was a statistically 

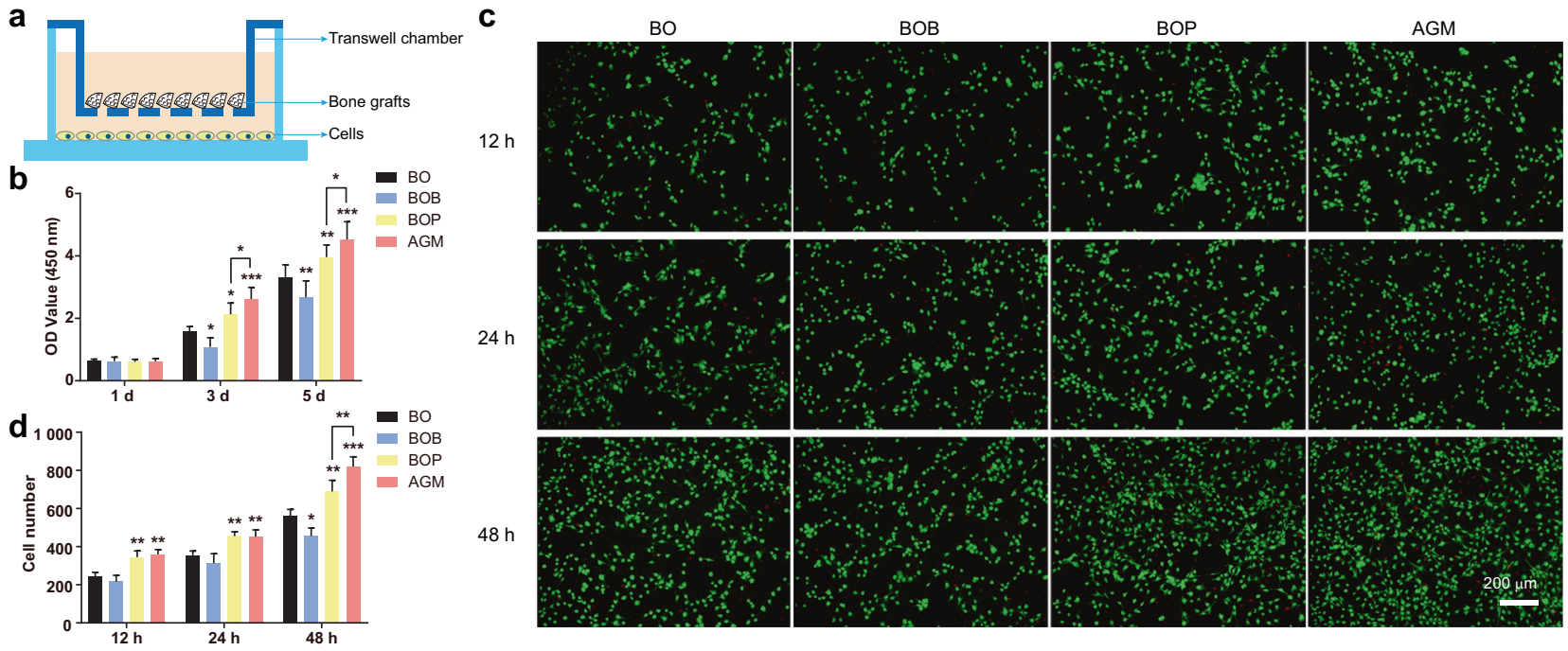

Fig. 3 AGM have better biocompatibility. a Schematic diagram of cell culture strategy. b CCK-8 assay on cell proliferation at 1, 3, and 5 days. c Fluorescence microscopy images of BMSCs incubated with bone grafts after staining with Calcein-AM/PI dyes. d Quantification of living cell number in c (scale bar $\left.=200 \mu \mathrm{m}, n=3,{ }^{* * *} P<0.001,{ }^{* *} P<0.01,{ }^{*} P<0.05\right)$

significant increase in the proliferation of rat BMSCs in the AGM group compared with the other groups (Fig. 3b). We also performed living (green) and dead (red) cell fluorescence staining at different time points of co-culture and found that the number of living cells in the PRF-containing groups was significantly higher than that in the non-PRF-containing groups during the same period, which is consistent with the CCK-8 test results (Fig. 3c, d). Besides, only minimal dead and apoptotic cells were observed during the 5-day culture (Fig. 3c). These results suggest that PRF has good biocompatibility and can accelerate cell proliferation in the current culture system, whereas high concentrations of BMP-2 inhibited the proliferation of BMSCs. What is most important, AGM can retain BMP-2 in the fibrin network so that the BMP-2 can be released continuously for some time, thereby promoting cell proliferation.

Transwell migration assay and cell scratch wound-healing assay were used to determine the effects of four grafts on the chemotaxis of rat BMSCs. Compared with the control group, the cells in the BOB and the BOP groups migrated to the scratch area more. Besides, the cells migrated more significantly to the scratch area in the AGM group than in the BOB and BOP groups (Fig. 4a, b). Quantitative analysis of migrated cells using the transwell assay further confirmed the positive effects of AGM on cell migration (Fig. 4c-e).

AGM promotes BMSCs mineralization in vitro

Alkaline phosphatase (ALP), a ubiquitous intracellular enzyme, is an indicator of the function and differentiation of osteoblasts; it is widely used in the study of abnormal bone metabolism and bone diseases and the detection of the degree of osteogenic differentiation $^{39}$. To detect the osteogenesis effect of AGM in vitro, we assessed the ALP staining of rat BMSCs co-cultured with these four grafts on the 7th day of mineralization (Fig. 3a). Results showed that the three other groups showed better osteogenic effects than Bio-Oss alone, with the group treated with AGM providing the strongest osteogenic effect (Fig. 5a). Alizarin Red Staining and quantification showed the same results (Fig. 5b, c). Interestingly, PRF showed a similar strength of osteogenesis effect to BMP-2 possibly because of the release of endogenous growth factors in PRF ${ }^{40}$. To evaluate the changes of BMSCs at the gene level during osteogenesis induced by PRF and BMP-2, we measured the expression levels of osteogenic differentiation-related genes including ALP, COL1A1, and RUNX2.
Results showed that both PRF and BMP-2 upregulated the expression of osteogenic differentiation-related genes, but no statistical difference was found between them, and the combined application could achieve a better osteogenesis effect (Fig. 5d).

The regeneration effect of AGM in the bone defect in vivo To further confirm the osteogenesis ability of AGM in vivo, we prepared $5 \mathrm{~mm}$ critical-sized bone defects in the bilateral parietal of rats and repaired them using the corresponding bone grafts. After 8 weeks, micro-CT showed that a small new bone formation in the Bio-Oss group, whereas the other groups showed significant osteogenic effects (Fig. 6a). Notably, new bone formation was pronounced for AGM, with corresponding quantitative data showing a $>40 \%$ ratio of bone to tissue in the defect area (Fig. 6b). Furthermore, bone substitutes moved around in the two groups without PRF ( $\mathrm{BO}$ and $\mathrm{BOB}$ ), whereas those in the other groups with PRF (BOP and AGM) were confined in the bone defect (Fig. 6a). Histological evaluation of $\mathrm{HE}$ staining can provide additional information about bone regeneration in the defect area (Fig. 6c). At 8 weeks after the surgery, HE staining showed that the tissue defect diameter was larger in the Bio-Oss group, whereas that in the three other groups, especially in AGM, was reduced to a certain extent, indicating rapid bone formation (Fig. $6 \mathrm{C}$ ). These results suggest that PRF and BMP-2 exert synergistic effects on bone remodeling and new bone formation. And a hypothetical scheme of osteogenesis of AGM is proposed basing on the above results (Fig. 7).

\section{DISCUSSION}

Sustained release of growth factors to the surroundings is one of the goals of tissue engineering. In this study, bone grafts without PRF were found to release a high concentration of BMP-2 at an early stage, but this was not what we expected. On the one hand, fast-released growth factors are easily metabolized by the human body, preventing them from playing a long-term stabilizing role in promoting osteogenesis ${ }^{21}$. On the other hand, the explosive release of growth factors such as BMP-2 can cause high local concentrations, leading to side effects such as bone resorption, local inflammation, postoperative fever and bleeding, and even cancer $^{41,42}$.

Unlike BOB which releases BMP-2 explosively, AGM releases BMP-2 slowly at a lower concentration. Studies have shown that 
a

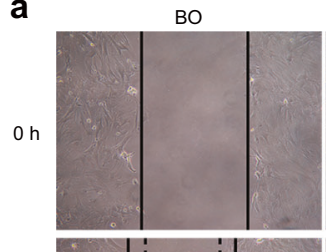

$24 \mathrm{~h}$
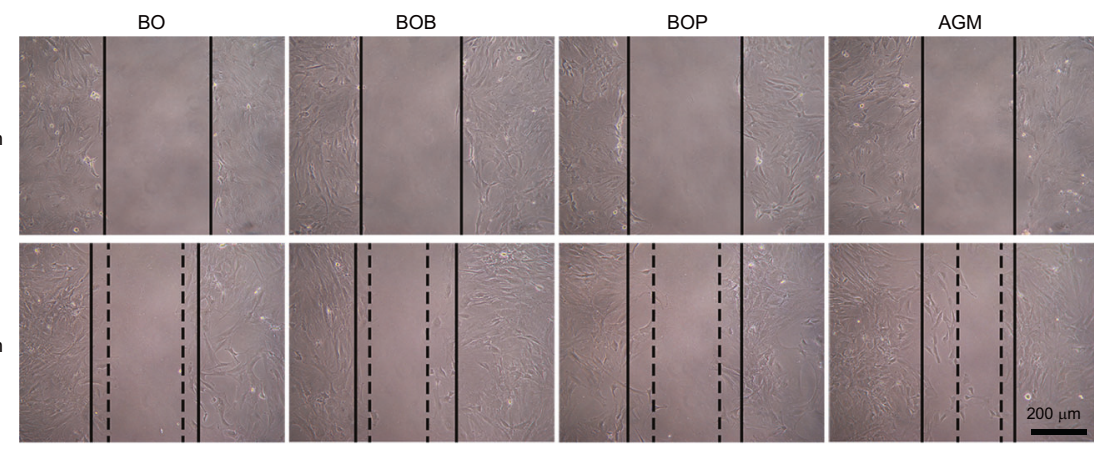

d

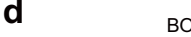

$\mathrm{BO}$

BOB

BOP

AGM

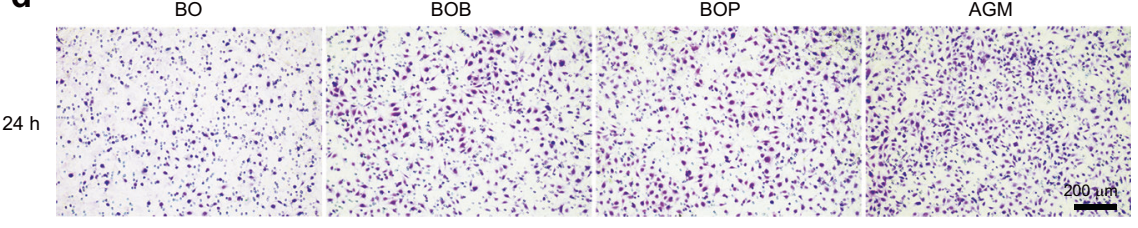

b

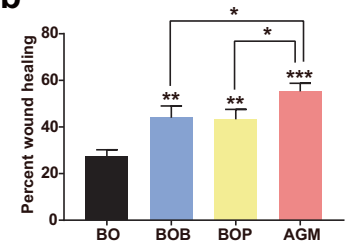

C

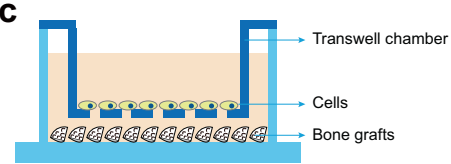

e

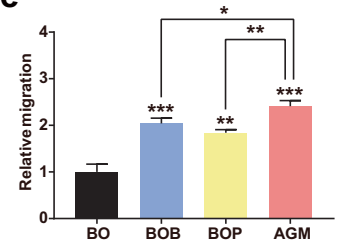

Fig. 4 AGM has a stronger recruitment function on BMSCs. a Representative microscopic images of wound healing at the beginning and after incubated with bone grafts for $24 \mathrm{~h}$. b Percent of wound healing was calculated as the ratio of the remaining scratch gap at $24 \mathrm{~h}$ and the original gap at $\mathrm{Oh}$. c Schematic diagram of cell culture strategy for transwell assay. d Representative microscopic images of BMSCs that migrated through the transwell in the migration assay. e The relative migration of cells compared to $\mathrm{BO}$ group in $\mathbf{d}\left(n=3,{ }^{* * *} P<0.001,{ }^{* *} P<\right.$ $\left.0.01,{ }^{*} P<0.05\right)$

high concentrations of BMP-2 can inhibit the proliferation of human primary periosteal cells, which plays an important role in fracture healing ${ }^{38}$. And lower concentrations of BMP-2 can promote the proliferation of chondrocytes ${ }^{43}$. Therefore, it is assumed that BOP explosively releases a high concentration of BMP-2 to inhibit cell proliferation. In contrast, with the sustained release of a low concentration of BMP-2 in AGM, the effect of promoting proliferation emerged. Besides, many studies have shown that BMP-2 composite delivery materials can promote the proliferation and osteogenic differentiation of osteoblasts at the same time ${ }^{44-47}$.

The famous "PASS" principle for successful guided bone regeneration surgery stands for space creation and maintenance, angiogenesis, primary wound closure, and stability of blood clots to allow regenerative tissue to grow in ${ }^{48}$. Therefore, bone grafts should be used for spatial maintenance during bone healing. However, in clinical treatment, the morphology of bone graft is hard to fit the defect unless titanium mesh or bone nails are used to fix the graft, leading to unstable osteogenic effect ${ }^{49}$.

Autografts are the gold standard for bone regeneration since they provide osteogenic cells, osteoinductive growth factors, and an osteoconductive scaffold, all essential for new bone formation $^{50,51}$. However, autografts have brought several disadvantages because of the high absorptivity and complication. The AGM prepared by Bio-Oss, PRF and BMP-2, was rich in bone substitutes with a low substitution rate, which could maintain the space of bone defect for a long time and play the role of osteoconduction. The fibrin scaffolds inside PRF wrapped around the bone graft, giving it initial stability similar to that of autograft. On the other hand, it has been reported that fibrin alone can play a role in provisional cell invasion and tissue regeneration ${ }^{26}$. Besides, integrated the effect of BMP-2, AGM simulated the characteristics of autograft in many aspects including osteoconduction and osteoinduction, which provided the potential possibility to mimic autograft in the clinic.

In the present study, we surprisingly discovered that xenograft modified by PRF has good operability and is easy to adjust to the shape of the bone defect. Heterogeneous bone grafts are of great clinical significance because they can prevent micromotion, minimize bone loss, and reduce the use of bone grafts and titanium meshes while maintaining space.

As mentioned above, Bio-Oss ${ }^{\varpi}$, a commonly used bone scaffold material in clinical practice, plays an osteoconductive role and maintains the space of the defect area, while BMP-2 plays an osteoinductive role and promotes cell migration ${ }^{21,37,52}$. PRF extracted from blood is rich in various cells including platelets and a variety of cytokines, such as TGF- $\beta 1$, PDGF, epidermal growth factor, and IGF, which play an important role in cell proliferation and migration ${ }^{53}$. PRF also contains a large number of leukocytes, which play a vital role in wound healing ${ }^{54}$. Leukocytes fight infection and regulate immunity by secreting key immunomodulatory cytokines such as interleukin$1 \beta$ (IL-1 $\beta$ ), IL-6, IL-4, and tumor necrosis factor-a to promote tissue healing ${ }^{5-58}$. The fibrin network in the PRF not only combines the entire bone scaffold to continuously release cytokines in the form of a hydrogel but also acts as a scaffold for cell migration, proliferation, and differentiation, providing a matrix for tissue reconstruction and accelerated mineralization ${ }^{26,34}$. In one-word, multiple factors of AGM jointly improve the effect of bone tissue regeneration.

Available bone grafts denote a wide range of materials, including allograft, xenograft, and artificial bone material ${ }^{59}$. In daily clinical applications, it is more than critical to select proper material depending on patient conditions. Implantation of the mix of autologous bone fragments and bone substitutes into the defect also showed an excellent osteogenic effect ${ }^{60}$. Drugs for topical application can also be personalized, and a variety of growth factors and cells can be used to promote osteogenesis and angiogenesis. In any case, it is crucial to combine bone substitutes and drugs through delivery materials. This study overcomes the secondary trauma of autologous transplantation, the inconvenience of xenotransplantation, and the explosive release of traditional BMP-2. Further, the bone graft proposed in this study partly compensates for the deficiencies of autograft and xenograft while maximizing their advantages, thereby providing practical guidance for the repair and treatment of clinical hard tissue defect. Moreover, this study not only fills the gap in current biological research of bone regeneration but also lays a solid theoretical foundation for the research and development of materials in tissue engineering and proposes new ideas for further development. 


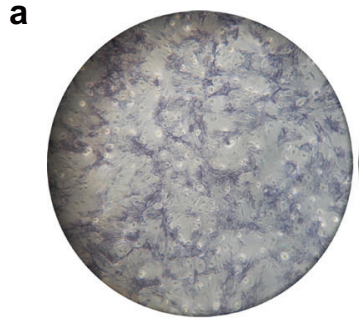

BO

b

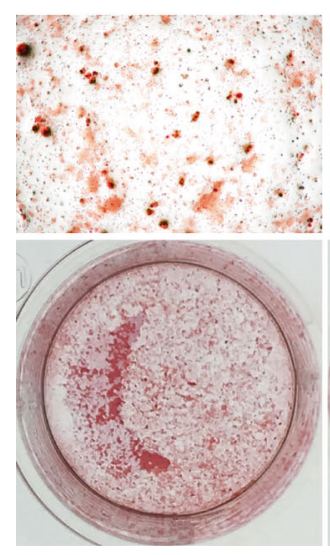

BO

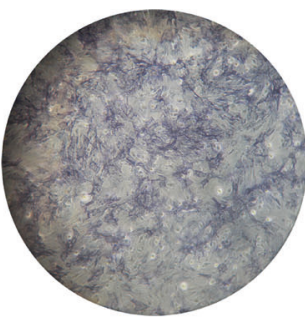

$\mathrm{BOB}$

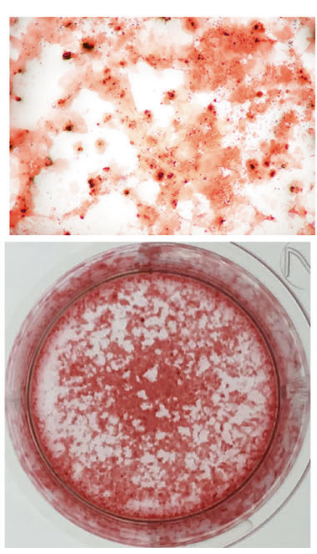

BOB

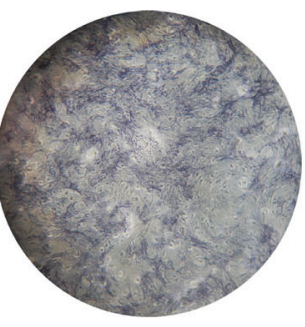

BOP

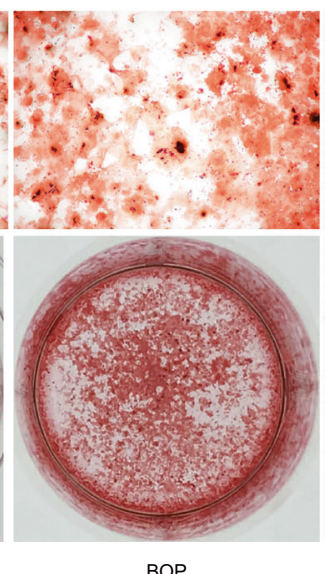

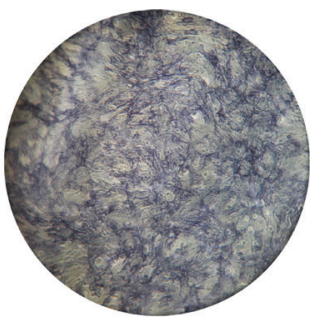

AGM

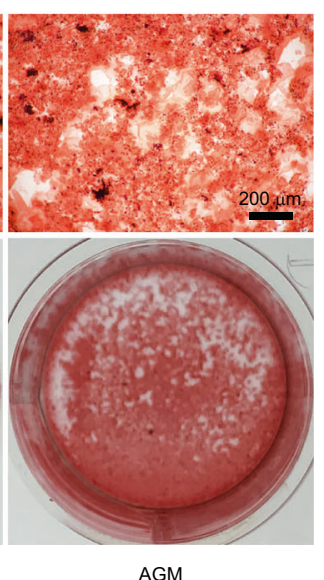

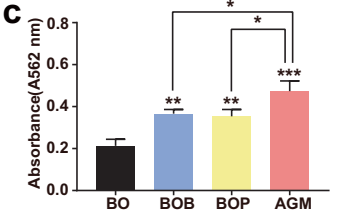
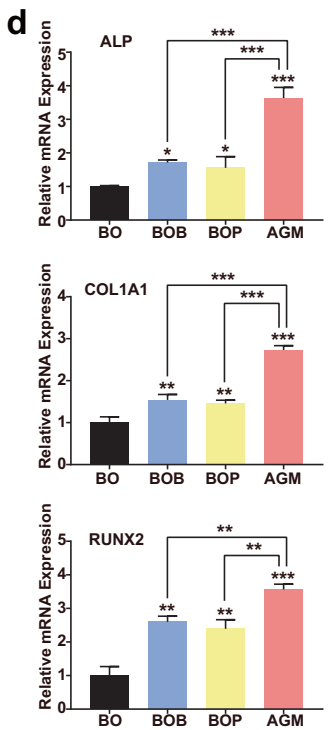

Fig. 5 AGM has a stronger mineralization promotion effect on BMSCs. a ALP staining of BMSCs incubated with the corresponding bone grafts for 7 days. b ARS staining of BMSCs incubated with the corresponding bone grafts for 14 days. $\mathbf{c}$ Quantification of staining in $\mathbf{b}$. $\mathbf{d}$ Osteogenic related genes (ALP, COL1A1, and RUNX2) expression were measured to confirm the osteoblast differentiation of BMSCs incubated with the corresponding bone grafts $\left(n=3,{ }^{* * *} P<0.001,{ }^{* *} P<0.01,{ }^{*} P<0.05\right)$

\section{CONCLUSIONS}

In this study, BMP-2 and Bio-Oss ${ }^{\circledast}$ were selected and improved by PRF, an autologous blood-derived preparation. The new type of bone graft, AGM, is clinically available and inherits the superior osteoconductivity property of Bio-Oss and osteoinduction property of BMP-2. It features strong operability and allows the controlled release of BMP-2. We confirmed the sustained release of BMP-2 in AGM, ensuring the long-term stable function of BMP2. PRF and BMP-2 in AGM exerted synergistic effects in promoting the proliferation, migration, and osteogenic differentiation of BMSCs. Besides, the rat parietal defect model showed that $\mathrm{BOB}$ and BOP both improved the osteogenic performance of Bio-Oss, and Bio-Oss combined with BMP-2 and PRF further improved the osteogenic performance in vivo. To a certain extent, these results support that AGM can solve the deficiencies of autograft and xenograft, and show great potential in clinical applications. Thus, this study lays the foundation for the future development of tissue engineering and provides new ideas for the modification of inorganic biomaterials.

\section{MATERIALS AND METHODS}

Preparation of PRF and bone grafts

For in vitro experiments, $8-10 \mathrm{~mL}$ of whole blood was obtained from the heart of a deeply anesthetized rat by cardiac puncture. The blood was then collected in the sterile vacuum tube (Kangjian Medical, Jiangsu, China) without anticoagulant and centrifuged immediately at room temperature at $700 \mathrm{r} \cdot \mathrm{min}^{-1}$ for 3 min (IntraLock, USA). PRF, the upper plasma layer, was collected for subsequent experiments. For in vivo experiments, $2 \mathrm{~mL}$ of whole blood was extracted through the jugular vein to prepare PRF. The bone substitute (Bio-Oss; ; Geistlich Biomaterials, Switzerland) and rat BMP-2 protein (Abbkine, California, USA) were used.
The experiment was divided into four groups: (1) Bio-Oss $(\mathrm{BO})$ : $0.25 \mathrm{~g}$ Bio-Oss $+0.2 \mathrm{~mL}$ phosphate-buffered saline (PBS), (2) BioOss /BMP-2 (BOB): $0.25 \mathrm{~g}$ Bio-Oss ${ }^{\oplus}+2 \mu \mathrm{g} \mathrm{BMP}-2+0.2 \mathrm{~mL}$ PBS, (3) Bio-Oss /PRF (BOP): $0.25 \mathrm{~g}$ Bio-Oss ${ }^{\oplus}+0.2 \mathrm{~mL} \mathrm{PRF;} \mathrm{and} \mathrm{(4)} \mathrm{Bio-Oss} /$ PRF/BMP-2 (AGM): $0.25 \mathrm{~g}$ Bio-Oss $+2 \mu \mathrm{g} \mathrm{BMP-2}+0.2 \mathrm{~mL}$ PRF.

\section{Scanning electron microscopy}

The materials were fixed with $2.5 \%$ glutaraldehyde at $4{ }^{\circ} \mathrm{C}$ overnight. They were then rinsed thrice with PBS and doubledistilled water $\left(\mathrm{ddH}_{2} \mathrm{O}\right)$; successively dehydrated once with $30 \%$, $50 \%, 70 \%, 80 \%$, and $95 \%$ ethanol; and then dehydrated twice with $100 \%$ ethanol for 5 min each. Critical point drying and spray gold treatment were performed. The cross-section of each sample was observed by SEM (Zeiss SIGMA, Carl Zeiss AG, UK).

\section{Release of BMP-2 in vitro}

To determine the cumulative release of growth factors at $1 \mathrm{~h}, 6 \mathrm{~h}$, $12 \mathrm{~h}, 1 \mathrm{~d}, 3 \mathrm{~d}, 7 \mathrm{~d}$, and $14 \mathrm{~d}$, we placed the samples in vials containing $5 \mathrm{~mL}$ of a-minimum essential medium (a-MEM) (HyClone, Thermo, USA) at $37^{\circ} \mathrm{C}$ to release growth factors. At each time point, $0.5 \mathrm{~mL}$ of medium was collected and the same volume of a-MEM was supplemented. The release of BMP-2 was detected by enzyme-linked immunosorbent assay (ELISA) (Abcam, Cambridge, UK) according to the manufacturer's instructions. Briefly, we coated the wells with standards and test samples, and blocked the remaining protein-binding sites in the coated wells with $5 \%$ non-fat dry milk. We added and incubated the following components in sequence: goat biotinylated detection polyclonal antibody against BMP-2, avidin-biotinperoxidase complex, and TMB solution. After sufficient color development, we added stop solution to the wells and read the optical density at $450 \mathrm{~nm}$. Finally, we prepared a standard curve and interpolated the concentration of test samples from this standard curve. 
a

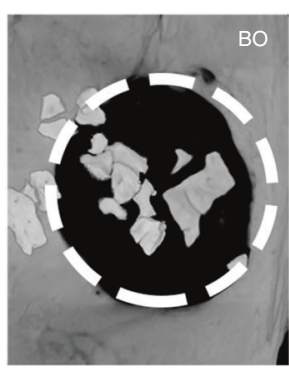

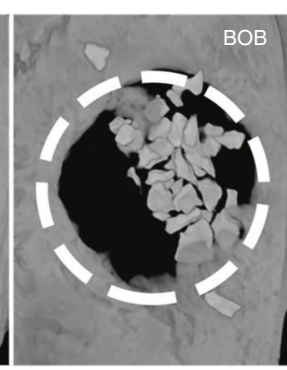
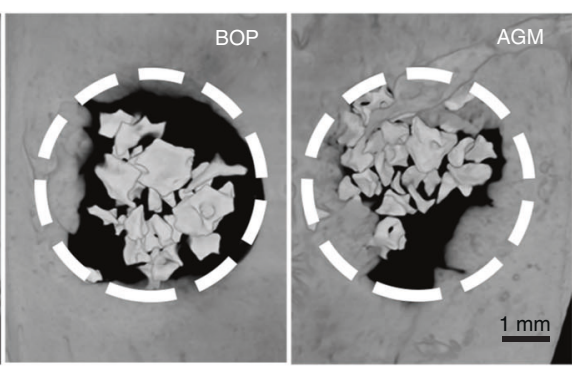

b

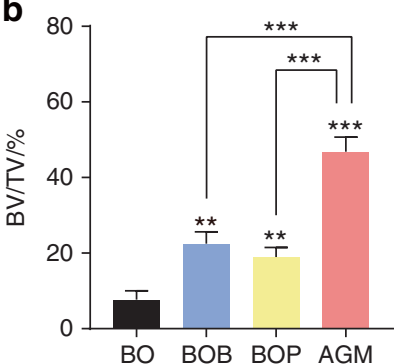

C

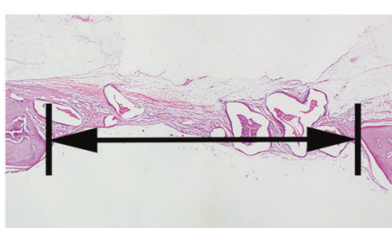

$\mathrm{BO}$

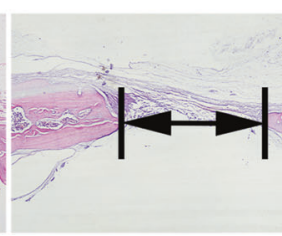

$\mathrm{BOB}$

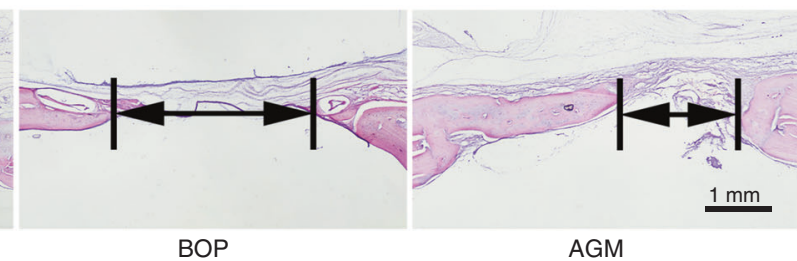

Fig. 6 AGM has a better repair effect of critical parietal defects in rats. a Three-dimensional (3D) images showing the different repair results in parietal defects assessed by micro-CT. b Micro-CT analysis of BV/TV in parietal defects. c Representative HE staining images of parietal defects at 8 weeks postoperatively (scale bar $=1 \mathrm{~mm}, n=3$, ***P<0.001, **P $<0.01$ )

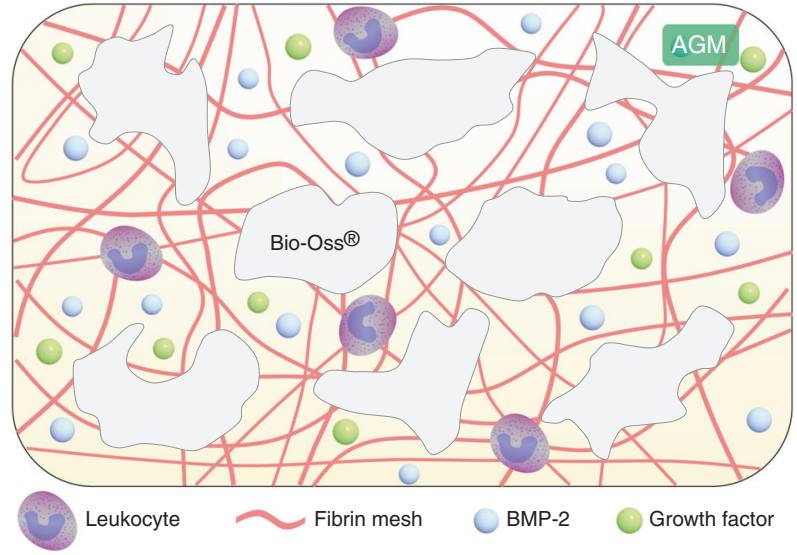

Fig. 7 Schematic illustration of the AGM. First, the bone substitutes are agglomerated by PRF, allowing convenient operation and promoting graft stability after implantation. Secondly, AGM promotes the long-term and stable release of BMP-2. Next, the fibrin mesh within PRF can serve as a scaffold for cell migration, proliferation, and differentiation, providing a matrix for tissue reconstruction. Finally, leukocytes and growth factors secreted by PRF also play important roles in anti-infection and immunoregulation

To determine the release of BMP- 2 at $1 \mathrm{~h}, 6 \mathrm{~h}, 12 \mathrm{~h}, 1 \mathrm{~d}, 3 \mathrm{~d}, 7 \mathrm{~d}$, and $14 \mathrm{~d}$, at each time point, we placed the samples in vials containing $5 \mathrm{~mL}$ of a-MEM at $37^{\circ} \mathrm{C}$ to release growth factors. At each time point, the culture solution was collected and $5 \mathrm{~mL}$ of fresh a-MEM was added. The release of BMP-2 was detected by ELISA (Abcam, Cambridge, UK).

Cell culture and osteogenic induction

The transwell chamber (Corning, USA) with $3 \mu \mathrm{m}$ pore membranes was selected for cell culture. The corresponding bone grafts were added in the upper chamber, and the lower chamber was used for cell culture. Rat BMSCs were harvested and incubated under standard cell culture conditions of $5 \% \mathrm{CO}_{2}$ at $37^{\circ} \mathrm{C}$ as previously reported ${ }^{61}$. The osteoblast inducing medium containing $50 \mu \mathrm{g} \cdot \mathrm{mL}^{-1}$ ascorbic acid (Sigma-Aldrich, USA), $10 \mathrm{nM}$ dexamethasone, and $10 \mathrm{mmol} \cdot \mathrm{L}^{-1} \beta$-glycerophosphate was used to replace the culture medium at $80 \%$ cell confluence. Cells were maintained under $5 \% \mathrm{CO}_{2}$ at $37^{\circ} \mathrm{C}$ with medium replacement every 3 days.
Cell proliferation determination using the Cell Counting Kit-8 (CCK-8) assay

The proliferation of rat BMSCs was evaluated by the CCK-8 (Beyotime, China) after 1,3 , and $5 \mathrm{~d}$ of culture with the corresponding bone grafts. In brief, rat BMSCs were seeded into a 96-well plate at the density of $5 \times 10^{3}$ cells per well. The solution containing $10 \mu \mathrm{L}$ of CCK- 8 original solution was used to replace the medium at 3 and 5 days. The cells were then incubated at $37^{\circ} \mathrm{C}$ for $1 \mathrm{~h}$. Absorbance at $450 \mathrm{~nm}$ was immediately quantified using a microplate reader (PowerWave XS2, BioTek, USA).

Assessment of cell vitality in vitro

The cells were seeded into a 12 -well plate with $3 \times 10^{5}$ cells per well. After culturing in the incubator for $6 \mathrm{~h}$, they were co-cultured with the corresponding bone grafts. Cells were collected at 12, 24, and $48 \mathrm{~h}$ for Calcein-AM/PI staining (YeaSen, Wuhan, China). Cells were first rinsed three times with $1 \times$ PBS and then incubated at $37^{\circ} \mathrm{C}$ in the dark for $20 \mathrm{~min}$ with $500 \mu \mathrm{L}$ of pre-prepared dye. Living/dead cells were detected and photographed under a fluorescence microscope using a 490 and $535 \mathrm{~nm}$ excitation filter, respectively (Olympus $\mathrm{rCo}$, Japan).

Cell scratch wound-healing assay

The cultured rat BMSCs were seeded in four six-well plates with $1 \times 10^{6}$ cells per well. When the cells reached the exponential growth stage, the cell density was about $90 \%$. A linear scratch was prepared in each well with a cell scraper, and these six-well plates were washed thrice with PBS to remove the subtracted cells. The cells were cultured with the corresponding culture medium in an incubator at $37^{\circ} \mathrm{C}$ with $5 \% \mathrm{CO}_{2}$. Sampling and taking photos were performed after culturing for $24 \mathrm{~h}$.

Transwell migration assays

Rat BMSCs were starved cultured with $2 \%$ fetal bovine serum (FBS) medium for $12 \mathrm{~h}$ and then seeded in a $12 \mathrm{~mm}$ transwell chamber with $1 \times 10^{4}$ cells. The cells resuspended in a-MEM without FBS were added to the upper chamber, and each conventional culture medium containing the corresponding bone graft was added to the lower chamber. The cells were cultured in an incubator for $24 \mathrm{~h}$ and fixed in $4 \%$ formaldehyde for $15 \mathrm{~min}$, stained with crystal violet (Beyotime, China), and washed with PBS three times before photographing. 


\begin{tabular}{|lll|}
\hline Table 1. & Primers used for real-time RT-PCR \\
\hline Name & Direction & Sequence $\left(5^{\prime}-3^{\prime}\right)$ \\
\hline ALP & Forward & TGGACGGTGAACGGGAAAAT \\
& Reverse & TAGTTCTGCTCATGGACGCC \\
RUNX2 & Forward & CCCAGTATGAGAGTAGGTGTCC \\
& Reverse & GGGTAAGACTGGTCATAGGACC \\
COL1A1 & Forward & TGGCAAAGACGGACTCAAC \\
& Reverse & GGCAGGAAGCTGAAGTCATAA \\
GAPDH & Forward & GCACCGTCAAGGCTGAGAAC \\
& Reverse & TGGTGAAGACGCCAGTGGA \\
\hline
\end{tabular}

ALP staining and Alizarin red S (ARS) staining

The level of mineralization of the extracellular matrix was determined to ALP staining and ARS staining in vitro.

Rat BMMSCs were seeded in 24-well plates at a density of $1 \times 10^{5}$ cells/well and cultured in an osteogenic medium. Rat BMSCs were washed with PBS thrice and fixed in 4\% paraformaldehyde for $20 \mathrm{~min}$ at day 7 during the osteogenic differentiation. The cells were then stained using the ALP kit (Beyotime, China) following the manufacturer's protocol. ARS staining was performed on day 14. Following $20 \mathrm{~min}$ of fixation in 4\% paraformaldehyde, cells were washed thrice with $\mathrm{ddH}_{2} \mathrm{O}$ and then stained with $0.1 \%$ ARS staining solution $(\mathrm{pH}$ 4.5) for $10 \mathrm{~min}$ at $37^{\circ} \mathrm{C}$. Finally, the cells were rinsed gently with $\mathrm{ddH}_{2} \mathrm{O}$ to terminate the reaction. The mineralized nodules were photographed under an optical microscope. The nodules were dissolved by $10 \%$ cetylpyridinium chloride for $2 \mathrm{~h}$. The absorbance at $562 \mathrm{~nm}$ represented the quantification of the ARS staining.

Quantitative real-time PCR (RT-PCR)

Total RNA of BMSCs was isolated with Trizol reagent (Invitrogen, USA). Total RNA $(1 \mu \mathrm{g})$ was used to synthesize complementary DNA using the PrimeScript RT reagent Kit (TaKaRa, Japan) as per the manufacturer's instruction. The primer sequences used in the present study are listed in Table 1. The data were normalized to glyceraldehyde-3-phosphate dehydrogenase expression and analyzed using the comparison $\mathrm{Ct}\left(2^{-\Delta \Delta \mathrm{Ct}}\right)$ method.

Ethical approval and experiments in vivo

All rats utilized in the study were purchased from the Centre for Disease Control of the province of Hubei, China. The experiment was performed following the policies requirement of the Ethics Committee for Animal Research, Wuhan University, China. The study protocol was approved by the Ethics Committee for Animal Use of the Institute of Biomedical Sciences (Protocol number 69/ 2017). Female SD rats aged 8 weeks used a trephine drill with a diameter of $5 \mathrm{~mm}$ to prepare to penetrate critical-size defects in both parietal bones. Bio-Oss ${ }^{\circ}$ with different treatments was filled in the bone defects. After 8 weeks, the parietal bones were collected and fixed with $4 \%$ paraformaldehyde for $48 \mathrm{~h}$ at $4{ }^{\circ} \mathrm{C}$ for Micro-CT, and an indicator of bone volume/tissue volume (BV/TV) was determined to evaluate bone mass (SCANCO MEDICAL mCT50, Switzerland). The samples were processed by $10 \%$ ethylenediaminetetraacetic acid, which was changed every 3 days for 3 months for decalcification. The difference of newly formed bone among the four groups was compared using hematoxylin-eosin (HE) staining under the manufacturer's direction after dehydration and paraffin embedding.

Statistical analysis

Data analysis was performed using GraphPad Prism software 6.0. Differences between the two groups were evaluated through two-way ANOVA and Student's $t$-test. All data are presented as mean \pm SD. At least three repeats are contained for each experiment. Statistical significance was considered at $* P<0.05$, ${ }^{* *} P<0.01,{ }^{* *} P<0.001$.

\section{DATA AVAILABILITY}

Research data are not shared.

\section{ACKNOWLEDGEMENTS}

This work was supported by the National Natural Science Foundation of China (Grant No. 82071095, 31570982).

\section{AUTHOR CONTRIBUTIONS}

Y.W. and H. Xia designed the research. G.Z. and Y.W. performed most of the experiments. C.Y., Q.Z., and H. Xu helped conduct some in vivo experiments. Y.Z. and X.Z. were involved in the revision of the manuscript. J.W. and J.Z. provided guidance for some in vivo experiments. Y.W. and G.Z. analyzed the results and wrote the manuscript.

\section{ADDITIONAL INFORMATION}

Supplementary information The online version contains supplementary material available at https://doi.org/10.1038/s41368-021-00120-w.

Competing interests: The authors declare no competing interests.

\section{REFERENCES}

1. Dang, W. et al. A bifunctional scaffold with CuFeSe2 nanocrystals for tumor therapy and bone reconstruction. Biomaterials 160, 92-106 (2018).

2. Park, J. H. et al. A rational tissue engineering strategy based on three-dimensional (3D) printing for extensive circumferential tracheal reconstruction. Biomaterials 185, 276-283 (2018).

3. Cui, Z. K. et al. Microporous methacrylated glycol chitosan-montmorillonite nanocomposite hydrogel for bone tissue engineering. Nat. Commun. 10, 3523 (2019).

4. Lin, S. et al. A magnesium-enriched $3 D$ culture system that mimics the bone development microenvironment for vascularized bone regeneration. Adv. Sci. (Weinh.) 6, 1900209 (2019).

5. Liu, S. et al. A cancellous bone matrix system with specific mineralisation degrees for mesenchymal stem cell differentiation and bone regeneration. Biomater. Sci. 7, 2452-2467 (2019).

6. Sanz, M. et al. Biomaterials and regenerative technologies used in bone regeneration in the craniomaxillofacial region: consensus report of group 2 of the 15th European Workshop on Periodontology on Bone Regeneration. J. Clin. Periodontol. 46(Suppl 21), 82-91 (2019).

7. Ikuta, K. et al. Predictors of complications in heat-treated autograft reconstruction after intercalary resection for malignant musculoskeletal tumors of the extremity. J. Surg. Oncol. 117, 1469-1478 (2018).

8. Brunello, G. et al. Powder-based 3D printing for bone tissue engineering. Biotechnol. Adv. 34, 740-753 (2016).

9. Fillingham, Y. \& Jacobs, J. Bone grafts and their substitutes. Bone Jt. J. 98-B, 6-9 (2016).

10. Othman, Z. et al. The role of ENPP1/PC-1 in osteoinduction by calcium phosphate ceramics. Biomaterials 210, 12-24 (2019).

11. Scarano, A. et al. Three-dimensional architecture and mechanical properties of bovine bone mixed with autologous platelet liquid, blood, or physiological water: an in vitro study. Int. J. Mol. Sci. 19, (2018).

12. Hyun, S. Y., Lee, J. H., Kang, K. J. \& Jang, Y. J. Effect of FGF-2, TGF-beta-1, and BMPs on teno/ligamentogenesis and osteo/cementogenesis of human periodontal ligament stem cells. Mol. Cells 40, 550-557 (2017).

13. Turri, A. et al. Guided bone regeneration is promoted by the molecular events in the membrane compartment. Biomaterials 84, 167-183 (2016).

14. Lu, C. H., Chang, Y. H., Lin, S. Y., Li, K. C. \& Hu, Y. C. Recent progresses in gene delivery-based bone tissue engineering. Biotechnol. Adv. 31, 1695-1706 (2013).

15. James, A. W. et al. A review of the clinical side effects of bone morphogenetic protein-2. Tissue Eng. Part B Rev. 22, 284-297 (2016).

16. Lutolf, M. P. et al. Repair of bone defects using synthetic mimetics of collagenous extracellular matrices. Nat. Biotechnol. 21, 513-518 (2003).

17. Tan, J. et al. Sustained release of two bioactive factors from supramolecular hydrogel promotes periodontal bone regeneration. ACS Nano 13, 5616-5622 (2019). 
18. Lee, G. H., Makkar, P., Paul, K. \& Lee, B. Incorporation of BMP-2 loaded collagen conjugated BCP granules in calcium phosphate cement based injectable bone substitutes for improved bone regeneration. Mater. Sci. Eng. C Mater. Biol. Appl. 77, 713-724 (2017).

19. Schmitt, C. et al. Bio-Oss(R) blocks combined with BMP-2 and VEGF for the regeneration of bony defects and vertical augmentation. Clin. Oral. Implants Res. 24, 450-460 (2013).

20. Luo, C. et al. Biomimetic open porous structured core-shell microtissue with enhanced mechanical properties for bottom-up bone tissue engineering. Theranostics 9, 4663-4677 (2019).

21. Boerckel, J. D. et al. Effects of protein dose and delivery system on BMP-mediated bone regeneration. Biomaterials 32, 5241-5251 (2011).

22. Krishnan, L. et al. Delivery vehicle effects on bone regeneration and heterotopic ossification induced by high dose BMP-2. Acta Biomater. 49, 101-112 (2017).

23. Miron, R. J. et al. A novel method for evaluating and quantifying cell types in platelet rich fibrin and an introduction to horizontal centrifugation. J. Biomed. Mater. Res. A 107, 2257-2271 (2019).

24. Engler-Pinto, A. et al. Effects of leukocyte- and platelet-rich fibrin associated or not with bovine bone graft on the healing of bone defects in rats with osteoporosis induced by ovariectomy. Clin. Oral Implants Res., https://doi.org/10.1111/ clr.13503 (2019).

25. Bhattacharjee, R., Sawatkar, G. \& Vinay, K. Platelet-rich fibrin dressings in treating nonhealing leg ulcers. J. Am. Acad. Dermatol. 80, e31-e32 (2019).

26. Nguyen, L. H. et al. Vascularized bone tissue engineering: approaches for potential improvement. Tissue Eng. Part B Rev. 18, 363-382 (2012).

27. Eren, G., Gürkan, A., Atmaca, H., Dönmez, A. \& Atilla, G. Effect of centrifugation time on growth factor and MMP release of an experimental platelet-rich fibrintype product. Platelets 27, 427-432 (2016).

28. He, L., Lin, Y., Hu, X., Zhang, Y. \& Wu, H. A comparative study of platelet-rich fibrin (PRF) and platelet-rich plasma (PRP) on the effect of proliferation and differentiation of rat osteoblasts in vitro. Oral. Surg. Oral. Med Oral. Pathol. Oral. Radio. Endod. 108, 707-713 (2009).

29. Dohan Ehrenfest, D. M., de Peppo, G. M., Doglioli, P. \& Sammartino, G. Slow release of growth factors and thrombospondin-1 in Choukroun's platelet-rich fibrin (PRF): a gold standard to achieve for all surgical platelet concentrates technologies. Growth Factors 27, 63-69 (2009).

30. Lundquist, R., Dziegiel, M. H. \& Agren, M. S. Bioactivity and stability of endogenous fibrogenic factors in platelet-rich fibrin. Wound Repair Regen. 16, 356-363 (2008).

31. Dohan Ehrenfest, D. M. et al. The impact of the centrifuge characteristics and centrifugation protocols on the cells, growth factors, and fibrin architecture of a leukocyte- and platelet-rich fibrin (L-PRF) clot and membrane. Platelets 29, 171-184 (2018)

32. Pelaez, M. et al. Effect of rhBMP-2 dose on bone formation/maturation in a rat critical-size calvarial defect model. J. Clin. Periodontol. 41, 827-836 (2014).

33. Wend, $\mathrm{S}$. et al. Reduction of the relative centrifugal force influences cell number and growth factor release within injectable PRF-based matrices. J. Mater. Sci. Mater. Med 28, 188 (2017).

34. Dohan, D. M. et al. Platelet-rich fibrin (PRF): a second-generation platelet concentrate. Part II: platelet-related biologic features. Oral. Surg. Oral. Med Oral. Pathol. Oral. Radio. Endod. 101, e45-50 (2006).

35. Wong, C. C. et al. Single-stage cartilage repair using platelet-rich fibrin scaffolds with autologous cartilaginous grafts. Am. J. Sports Med. 45, 3128-3142 (2017).

36. Steller, D., Herbst, N., Pries, R., Juhl, D. \& Hakim, S. G. Positive impact of platelet-rich plasma and platelet-rich fibrin on viability, migration and proliferation of osteoblasts and fibroblasts treated with zoledronic acid. Sci. Rep. 9, 8310 (2019).

37. Mayr-Wohlfart, U. et al. Vascular endothelial growth factor stimulates chemotactic migration of primary human osteoblasts. Bone 30, 472-477 (2002).

38. Kim, H. K., Oxendine, I. \& Kamiya, N. High-concentration of BMP2 reduces cell proliferation and increases apoptosis via DKK1 and SOST in human primary periosteal cells. Bone 54, 141-150 (2013).

39. Demers, L. M., Costa, L. \& Lipton, A. Biochemical markers and skeletal metastases. Cancer 88, 2919-2926 (2000)

40. Nurden, A. T. Platelets, inflammation and tissue regeneration. Thromb. Haemost. 105(Suppl 1), S13-33 (2011).

41. Mesfin, A. et al. High-dose rhBMP-2 for adults: major and minor complications: a study of 502 spine cases. J. Bone Jt. Surg. Am. 95, 1546-1553 (2013).
42. Martino, M. M., Briquez, P. S., Maruyama, K. \& Hubbell, J. A. Extracellular matrixinspired growth factor delivery systems for bone regeneration. Adv. Drug Deliv. Rev. 94, 41-52 (2015).

43. Shu, B. et al. BMP2, but not BMP4, is crucial for chondrocyte proliferation and maturation during endochondral bone development. J. Cell Sci. 124, 3428-3440 (2011).

44. Amirian, J., Linh, N. T., Min, Y. K. \& Lee, B. T. Bone formation of a porous gelatinpectin-biphasic calcium phosphate composite in presence of BMP-2 and VEGF. Int. J. Biol. Macromol. 76, 10-24 (2015).

45. Kim, S. E. et al. The effect of immobilization of heparin and bone morphogenic protein-2 (BMP-2) to titanium surfaces on inflammation and osteoblast function. Biomaterials 32, 366-373 (2011).

46. Zouani, O. F., Chollet, C., Guillotin, B. \& Durrieu, M. C. Differentiation of preosteoblast cells on poly(ethylene terephthalate) grafted with RGD and/or BMPs mimetic peptides. Biomaterials 31, 8245-8253 (2010).

47. van den Dolder, J., de Ruijter, A. J., Spauwen, P. H. \& Jansen, J. A. Observations on the effect of BMP-2 on rat bone marrow cells cultured on titanium substrates of different roughness. Biomaterials 24, 1853-1860 (2003).

48. Wang, H. L. \& Boyapati, L. "PASS" principles for predictable bone regeneration. Implant Dent. 15, 8-17 (2006).

49. Buser, D., Dula, K., Hess, D., Hirt, H. P. \& Belser, U. C. Localized ridge augmentation with autografts and barrier membranes. Periodontol 2000 19, 151-163 (1999).

50. Clafshenkel, W. P. et al. A novel calcium aluminate-melatonin scaffold enhances bone regeneration within a calvarial defect. J. Pineal Res 53, 206-218 (2012).

51. Raina, D. B. et al. A facile one-stage treatment of critical bone defects using a calcium sulfate/hydroxyapatite biomaterial providing spatiotemporal delivery of bone morphogenic protein-2 and zoledronic acid. Sci Adv. 6, https://doi.org/ 10.1126/sciadv.abc1779 (2020).

52. Duan, R. et al. Variation of the bone forming ability with the physicochemical properties of calcium phosphate bone substitutes. Biomater. Sci. 6, 136-145 (2017).

53. Becker, R. C., Sexton, T. \& Smyth, S. S. Translational implications of platelets as vascular first responders. Circ. Res. 122, 506-522 (2018)

54. Kawazoe, T. \& Kim, H. H. Tissue augmentation by white blood cell-containing platelet-rich plasma. Cell Transpl. 21, 601-607 (2012).

55. Rajendrakumar, S. K. et al. Peroxidase-mimicking nanoassembly mitigates lipopolysaccharide-induced endotoxemia and cognitive damage in the brain by impeding inflammatory signaling in macrophages. Nano Lett. 18, 6417-6426 (2018).

56. Eming, S. A., Brachvogel, B., Odorisio, T. \& Koch, M. Regulation of angiogenesis: wound healing as a model. Prog. Histochem. Cytochem. 42, 115-170 (2007).

57. Zhao, Q. et al. Near-infrared light-sensitive nano neuro-immune blocker capsule relieves pain and enhances the innate immune response for necrotizing infection. Nano Lett. 19, 5904-5914 (2019).

58. Zhang, P. et al. Fe3O4@TiO2-Laden neutrophils activate innate immunity via photosensitive reactive oxygen species release. Nano Lett. 20, 261-271 (2020).

59. Fernandez de Grado, G. et al. Bone substitutes: a review of their characteristics, clinical use, and perspectives for large bone defects management. J. Tissue Eng. 9, 2041731418776819 (2018).

60. Galindo-Moreno, P. et al. Histopathological comparison of healing after maxillary sinus augmentation using xenograft mixed with autogenous bone versus allograft mixed with autogenous bone. Clin. Oral. Implants Res. 29, 192-201 (2018).

61. Liu, S. et al. MSC transplantation improves osteopenia via epigenetic regulation of Notch signaling in lupus. Cell Metab. 22, 606-618 (2015).

(i) Open Access This article is licensed under a Creative Commons Attribution 4.0 International License, which permits use, sharing, adaptation, distribution and reproduction in any medium or format, as long as you give appropriate credit to the original author(s) and the source, provide a link to the Creative Commons license, and indicate if changes were made. The images or other third party material in this article are included in the article's Creative Commons license, unless indicated otherwise in a credit line to the material. If material is not included in the article's Creative Commons license and your intended use is not permitted by statutory regulation or exceeds the permitted use, you will need to obtain permission directly from the copyright holder. To view a copy of this license, visit http://creativecommons. org/licenses/by/4.0/.

(c) The Author(s) 2021 